\title{
Selective visual scaling of time-scale processes facilitates broadband learning of isometric force frequency tracking
}

\author{
Adam C. $\operatorname{King}^{1} \cdot \operatorname{Karl}$ M. Newell ${ }^{2}$ \\ Published online: 4 June 2015 \\ (C) The Psychonomic Society, Inc. 2015
}

\begin{abstract}
The experiment investigated the effect of selectively augmenting faster time scales of visual feedback information on the learning and transfer of continuous isometric force tracking tasks to test the generality of the self-organization of $1 / f$ properties of force output. Three experimental groups tracked an irregular target pattern either under a standard fixed gain condition or with selectively enhancement in the visual feedback display of intermediate $(4-8 \mathrm{~Hz})$ or high $(8-12 \mathrm{~Hz})$ frequency components of the force output. All groups reduced tracking error over practice, with the error lowest in the intermediate scaling condition followed by the high scaling and fixed gain conditions, respectively. Selective visual scaling induced persistent changes across the frequency spectrum, with the strongest effect in the intermediate scaling condition and positive transfer to novel feedback displays. The findings reveal an interdependence of the timescales in the learning and transfer of isometric force output frequency structures consistent with $1 /$ f process models of the time scales of motor output variability.
\end{abstract}

Keywords Motorlearning - Time scales · Visual information . Isometric force

When learning a novel motor skill, an individual has access to multiple sources of information derived from different

Adam C. King

aking@truman.edu

1 Health and Exercise Science Department, Truman State University, 2120 Pershing Building, Kirksville, MO 63501, USA

2 Department of Kinesiology, University of Georgia, G10 Aderhold Hall, Athens, GA 30602, USA modalities (e.g., vision, touch, and audition) to facilitate a change in motor performance as a function of practice. In addition to these inherent perceptual sources, augment information can be provided in the practice environment to guide performance toward the task goal. Two of the most effective categories of augmented information that enhance learning and performance are information about (a) the to-beproduced movement that is provided in advance of a trial (such as in instructions and demonstrations) and (b) feedback about the movement and its outcome of the just completed trial (Bilodeau \& Bilodeau, 1961; Hodges \& Franks, 2004; Kernodle \& Carlton, 1992; Newell, 1996; Newell, Morris, \& Scully, 1985). A core issue in motor learning is the effect of informational constraints on the learning, retention, and transfer of motor skills. The manipulation of different informational constraints present during acquisition includes the structure of the practice environment (e.g., variability of practice and contextual interference) as well augmented information (i.e., instructions, concurrent, and feedback) provided to the learner.

The manipulation of different forms of informational constraints on motor learning relates to varying theoretical interpretation. Traditional viewpoints posited that augmented information, such as knowledge of results or knowledge of performance, acts to strengthen the respective memory trace for action (Schmidt, 1975). However, performance degrades when the learner (a) becomes dependent on the augmented feedback (Armstrong, 1970) or (b) is unable to process inherent feedback relative to augmented information (Swinnen, Schmidt, Nicholson, \& Shapiro, 1990). Contemporary approaches related to the specificity of practice hypothesis propose that learning is specific to the dominant source of afferent information present during practice (Ernst \& Banks, 2002; Proteau, 1992; Tremblay \& Proteau, 1998). For example, an individual develops a highly specific internal sensorimotor 
representation of the movement pattern during practice to ensure optimal performance according to the dominant source of afferent information. A change in the dominant sensory source (i.e., vision to haptics or kinesthetic information) or in the reliability of sensory information (Ernst \& Banks, 2002) degrades performance - even following extensive amounts of practice (Proteau, Tremblay, \& Dejaeger, 1998).

An alternative theoretical viewpoint to the role of information on motor learning is the ecological perspective, which proposes that the manipulation of informational constraints can channel a learner to search for unique movement solutions within the perceptual-motor workspace (Bennett, Davids, \& Woodcock, 1999; Kugler, Kelso, \& Turvey, 1980; Newell, 1991). With practice the learner will identify task-relevant sources of perceptual information that support changes of performance and continue to develop a tight coupling between the mapping of information and movement dynamics in the workspace. In this view, information constraints extend beyond the typical preperformance (e.g., instructions) and postperformance (augmented feedback) variables to include concurrent forms of feedback that operate on multiple time scales to interact with the multiple dimensions of motor learning (Kugler et al., 1980; Newell, 1996; Studenka, King, \& Newell, 2014).

In the informational constraints approach, manipulation of different practice variables is proposed to induce exploratory movements that allow the learner to enhance the mapping between movement patterns and the demands of the task. For example, intermixing the luminance condition (i.e., normal and dark) during practice promotes enhanced learning of a catching skill in children, compared to a single luminance condition (Bennett et al., 1999; Whiting, Savelsbergh, \& Pijpers, 1995). Collectively, information acts as a constraint to action in confluence with the state of the individual and the specific context of the environment.

The role information has on an individual's search strategies requires an understanding of the potential movement solutions afforded by the task constraints, learners natural tendencies to search the workspace, and the use of augmented information (Newell, 1991). Practical and experimental challenges exist to implementing such an approach in discrete motor tasks where the temporal duration of the action is limited. Conversely, during continuous skills, such as visuomotor tracking and driving a car, information can be modulated in a number of different ways (e.g., delay, gain, intermittency), and these experimental manipulations have a strong influence on both motor performance outcome and organization of the movement pattern (Jagacinski \& Flach, 2003; Poulton, 1974).

Continuous tracking tasks also afford the investigation of multiple time-scale processes that relate to feedforward and feedback control mechanisms (Jagacinski \& Flach, 2003; Pew, 1974; Poulton, 1974). The output of continuous tracking can be decomposed into a set of frequency structures
(Bassingthwaighte, Liebovitch, \& West, 1994; Stanley \& Franks, 1990; Ward, 2002) that reveal the task-dependent interaction of slow and fast time-scale processes.

The structural spatial and temporal pattern of the force output operates over an effective bandwidth (out to $\sim 12 \mathrm{~Hz}$ ) of time scales and is consistent with the $1 / \mathrm{f}$-like processes of selforganization (Haken, 1983) and intercomponent (MayerKress, Deutsch, \& Newell, 2003; Van Orden, Holden, \& Turvey, 2003) dynamics. This organization in force output structure is dependent on the confluence of task, environment, and organismic constraints (Sosnoff, Valantine, \& Newell, 2009).

In the isometric force tracking task, the manipulation of the visual gain - the ratio of pixel representation to motor output is an informational constraint that enhances or degrades tracking performance dependent on the level of gain. For example, small increments of visual gain of the force output reduces tracking error, but beyond a critical gain value degrades performance (Newell \& McDonald, 1994; Sosnoff \& Newell, 2006). In the standard manipulation of visual gain, the visual display of the amplitude of motor output is uniformly enhanced over all frequency components by the same gain factor (Beuter, Haverkamp, Glass, \& Carrière, 1995; Jagacinski \& Flach, 2003; Newell \& McDonald, 1994; Sosnoff \& Newell, 2006). Therefore, the same relative amplitude contributions of slow and fast time scales are preserved in the force output. Consequently, the relative visual information of faster timescale processes may be a limiting informational constraint (reduced in the display) to force output adaptability and performance during isometric force tracking (Sosnoff \& Newell, 2008).

An individuals ability to adaptively reorganize faster timescale processes associated with prospective control (Studenka \& Newell, 2013) is in part influenced by the characteristic properties of low (i.e., large amplitude movements) and high (i.e., rapid, small amplitude oscillations) frequency components evident in the visual display. Experimentally, $\mathrm{Hu}$ and Newell (2010) introduced a manipulation that altered the relative visual feedback information of low- and high-frequency components of force output. Effectively, a selective scaling (i.e., gain) of visual information was applied to narrow bandwidths of frequency components (i.e., $4-8$ and $8-12 \mathrm{~Hz}$ ) of isometric force output to induce a change in the relative visual feedback information of shorter time-scale processes. The findings showed that under select scaling conditions individuals reduced tracking error and modulated shorter time-scale processes $(8-12 \mathrm{~Hz})$ in a task-dependent manner.

With respect to motor learning, several investigations have shown a persistent change of isometric force structure as a function of practice (Newell, Broderick, Deutsch, \& Slifkin, 2003; Sosnoff \& Voudrie, 2009); however, only recently have studies explored the effect of different practice conditions on the learning and transfer of isometric force control (King \& 
Newell, 2013; Studenka et al., 2014). The aim of present study was to examine the effect of different informational constraints on the learning and transfer of isometric force dynamics. Operationally, a manipulation similar to the selective scaling of visual feedback introduced by $\mathrm{Hu}$ and Newell (2010) was used to create different practice environments - fixed gain (Jagacinski \& Flach, 2003), enhanced scaling of intermediate $(4-8 \mathrm{~Hz})$ or high $(8-12 \mathrm{~Hz})$ frequency bandwidths, experienced by the participants. The central aim was to examine the interaction between practice and the scaling of selected frequency bandwidths of visual information on the learning and transfer of isometric force dynamics.

During the practice phase, the hypotheses investigated were that (a) reductions in tracking error as a function of practice would occur through task-dependent changes of force output structure independent of visual scaling (Sosnoff \& Voudrie, 2009); (b) the interaction between practice and visual scaling (e.g., intermediate and high scaling conditions) would enhance force-tracking performance by channeling individuals to use faster time scales of visual feedback information (Sosnoff \& Newell, 2006); and (c) the influence of the selective scaling of a narrow bandwidth of force output time scales would generalize to the facilitation of nonvisually enhanced frequency components of isometric force control.

Following extended practice, performance was examined in two transfer conditions that altered (enhanced or withdrawal of selective scaling manipulation) the properties of the visual feedback information compared to the practice environment. The specificity of practice hypothesis (Ernst \& Banks, 2002; Proteau, 1992) predicts a degradation of performance when the dominant source of afferent information used to obtain optimal performance accuracy is changed or withdrawn; however, it is unclear whether this hypothesis addresses changes in the specific properties of the dominant afferent information. The informational constraints approach proposes that practice under the different selective scaling conditions may facilitate the exploration and use of faster time scales of force output to enhance tracking performance. Thus, it is predicted that individuals who practice with augmented visual feedback information of shorter time scales will exhibit positive transfer compared to the fixed gain group.

\section{Method}

\section{Participants}

Twenty-two young adults ( 10 females, mean age $=24$ years $)$ who were naïve to the experimental manipulation volunteered to partake in this study. All participants were right-hand dominant, as determined by their preferred writing hand, and were free of any neuromuscular disorders or injuries to the upper limbs. Written informed consent that was approved by the
Pennsylvania State University Institutional Review Board was completed prior to participation.

\section{Apparatus}

An Entran ELFS-B3 load cell (Entran Devices, Inc., Fairfield, NJ) attached to a 16 bit A/D board (DT9804; Data Translation, Marlboro, MA) used to measure isometric abduction force output produced by the participant's right index finger. Force data were collected at a sample rate of $160 \mathrm{~Hz}$. Participants sat comfortably in front of a $43.2 \mathrm{~cm}$ LCD monitor (resolution $1280 \times 1040$ ) with arms placed in a prone position on the table. The participant's hand was placed with the distal interphalangeal joint of right index finger against the vertically fixed load cell. During trials, participants were instructed to maintain a fixed position of the fingers, hand, and forearm against the table. The experimental setup was similar to King and Newell (2013).

\section{Procedures}

Estimation of maximal voluntary contraction At the beginning of the experimental session, the participant's maximal voluntary contraction (MVC) was determined by producing isometric abduction force. Force was generated with the right index finger toward the midline of the body by pushing against a load. Each participant completed 3 MVC trials of $6 \mathrm{~s}$ with $20 \mathrm{~s}$ rest between trials. Each participant's MVC was defined by the highest force level achieved over the trials.

Experimental task and instructions During all trials, participants adjusted their force output to match a red target line displayed on the monitor and viewed online visual feedback of their performance displayed as a series of yellow dots. The force output moved left to right across the screen with time. The same target pattern was used for all practice and transfer trials (see Fig. 1). Each trial had a $20 \mathrm{~s}$ duration. The target pattern was characterized by a fractal dimension that consisted of frequency content from $0-12 \mathrm{~Hz}$ and exhibited a spectral slope $(\sim 1.5)$ between pink and brown noise. The wave form was constructed according to fractional Brownian motion (see King \& Newell, 2013; Sosnoff et al., 2009). The target line mean was matched to $10 \%$ of the participant's MVC, and the amplitude of the waveform was scaled to $\pm 5 \%$ of the participant's MVC.

A visually displayed feedback score was presented after each trial, and participants were instructed to reduce the score to as low a level as they could achieve as a function of practice. The score was the root mean square error (RMSE) between force output and target pattern and was calculated with the following equation: RMSE $=\left[\Sigma\left(s-f_{i}\right)^{2} /(n-1)\right]^{1 / 2}$, where $s$ is the value of the target, $f_{i}$ is the $i$ th force sample and $n$ is the number of data samples. 
Target Pattern

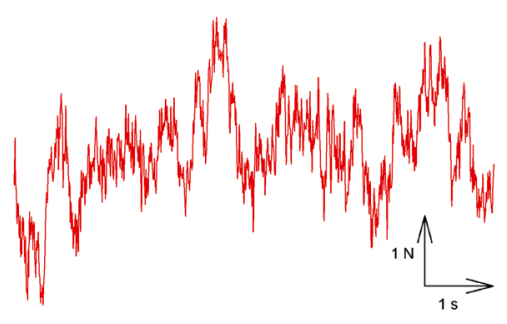

Intermediate Scaling

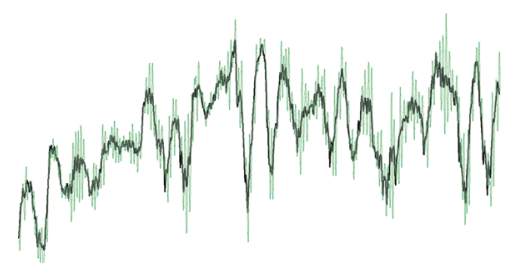

Fig. 1 Experimental condition examples. (top-left) Target pattern displayed to the participants. (top-right) Actual force output for a representative participant in the fixed gain condition. (bottom-left) and

Participants were assigned to one of three experimental groups defined by the visual scaling condition of the feedback information of force output (see Fig. 1). Two experimental groups, intermediate-frequency $(n=8)$ and high-frequency $(n=8)$ scaling, practiced the isometric task with an enhanced and selective visual scaling of $4-\mathrm{Hz}$ and $8-12 \mathrm{~Hz}$ frequency components, respectively. For example, the visual feedback display of the force output for the intermediate frequency scaling was manipulated by applying a gain factor to the middle frequency components (i.e., $4-8 \mathrm{~Hz}$ ) of the force output. The procedure of selectively scaling particular frequency components included the use of a band pass filter with a pass-band of 4-8 Hz applied to the force output. The filtered output was then multiplied by a predefined scaling factor (i.e., gain $=4$ ) and added to the original force output. The concatenated signal that included the original and scaled filtered force was visually displayed to the participants. The same procedure was applied in the high frequency scaling condition except that the band pass filter was applied to 8$12 \mathrm{~Hz}$ components. The force output was delayed approximately $60 \mathrm{~ms}$ for the $4-8 \mathrm{~Hz}$ and $8-12 \mathrm{~Hz}$ scaling conditions, and $30 \mathrm{~ms}$ for the fixed gain condition. These delays are shorter than those reported for minimal visual processing in similar tracking tasks (Slifkin, Vaillancourt, \& Newell, 2000).

A third group of participants $(n=6)$ served as a control in that these individuals practiced the task with a fixed gain factor of the visual feedback display of force output (see Fig. 1). The fixed gain factor was defined a priori and related to the ratio of pixel representation of force output that had previously been shown to approximate the production of best isometric force performance (Hong \& Newell,
Fixed Gain

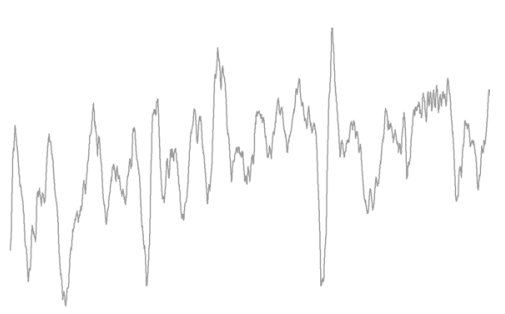

High Scaling

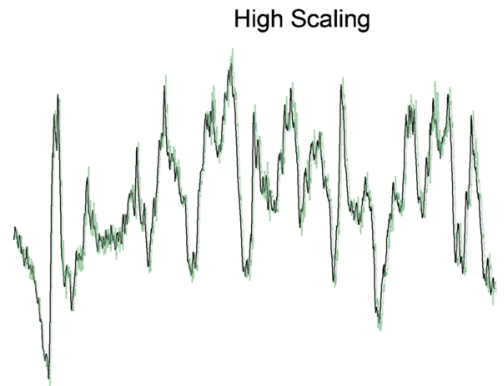

(bottom-right) Actual (dark line) and visually scaled (green line) feedback force trajectories for the intermediate (i.e., 4-8 Hz) and high (i.e., 8-12 Hz) selective scaling of feedback information. (Color figure online)

2008; Sosnoff \& Newell, 2006). The visual gain was 100 pixels per Newton $(\mathrm{p} / \mathrm{N})$ of force.

After 10 blocks of five trials in the practice phase, performance was examined in two transfer conditions: (a) a fixed gain test in which the test condition was the same as that practiced by the control group (i.e., experimentally defined fixed gain) and (b) a visually scaled test in which the test condition was the same as that practiced by the high-frequency scaling group (i.e., scaling of $8-12 \mathrm{~Hz}$ ). The order of the tests was counterbalanced with the constraint that two similar tests were not conducted consecutively. The transfer tests consisted of a block of five trials. The experimental procedures were repeated in the same manner over 2 consecutive days of practice.

\section{Data analysis}

For each trial, the initial $2 \mathrm{~s}$ and final $1 \mathrm{~s}$ of force data were removed to avoid the initial force stabilization or any premature cessation of force production. Data processing was performed with software written in MATLAB Version 7.0.

Task performance The force output was analyzed with the root mean square error (RMSE) to assess tracking error as a function of practice and transfer (see previous equation).

Structure of force output The structure of force output was analyzed in the frequency domain through a power spectral analysis. Custom-written software in MATLAB Version 7.0 was used to compute the power spectrum of the force output for each trial. Then, a linear regression was performed on the $\log -\log$ spectrum over a bandwidth that included $0-12 \mathrm{~Hz}$ 
with the DC component removed from the signal. The linear slope of the log power to log frequency from the spectral analysis provided an index of the change in power as a function of frequency.

Coherence analysis The coupling between the force output and target pattern was examined by performing a coherence analysis. Coherence provides the cross-correlation between two signals in the frequency domain. The output values range from 0 to 1 with higher values representing higher correlations. Coherence was computed using the minimum variance distortion response method with window length of 100 points and frequency resolution of $0.16 \mathrm{~Hz}$ (Benesty, Chen, Huang, $\&$ Member, 2005). Coherence between target and force output signals was computed over the $0-12 \mathrm{~Hz}$ range and the mean coherence of successive $2 \mathrm{~Hz}$ frequency bandwidths was analyzed.

\section{Statistical analysis}

To examine the interactive effects of visual scaling and practice the dependent variables were independently examined in separate three-way $(2 \times 10 \times 3)$ repeated measures analysis of variance (ANOVA) with day (2), block (10), and visual scaling group (3) as the main factors. The initial two factors were within-participant factors and feedback group was a betweenparticipant factor. Performance during the transfer tests was examined in a 2 (day) $\times 2$ (transfer condition) $\times 3$ (visual scaling group) mixed-model ANOVA.

Greenhouse-Geisser corrections were used in cases of violation of sphericity. Post hoc comparisons for the betweenparticipant factor were conducted using the Tukey Honestly Significant Difference method. All statistics were evaluated using SPSS (IBM), and significance was defined when there was less than a $5 \%$ chance $(p<.05)$ of making a Type I error.

\section{Results}

\section{Maximal force output}

The mean MVC over both practice days was $19.61 \mathrm{~N}(S D=$ 5.88). A 2 (practice day) $\times 3$ (visual scaling group) ANOVA revealed that MVC on day $1(M=19.74, S D=6.31)$ was not significantly different than on day $2(M=19.48, S D=5.56)$. There was not a statistically significant difference between the groups $(p=.75)$ on MVC values.

\section{Task performance: Practice}

Root mean square error (RMSE) Figure 2 illustrates the mean RMSE as a function of practice (block and day) for each visual scaling condition. There was a significant day $x$

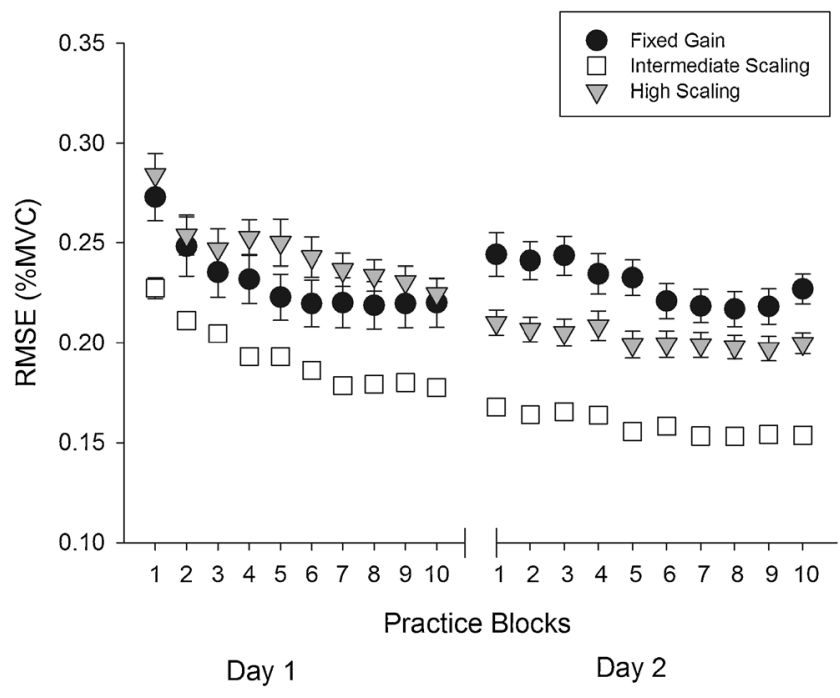

Fig. 2 RMSE as a function of practice and visual scaling conditions. Error bars represent standard error across participants

visual scaling group interaction, $F(2,19)=5.64, p .05$, $\eta_{\mathrm{p}}{ }^{2}=0.37$, as well as significant main effects of day, $F(1,19)=25.76, p<.001, \eta_{\mathrm{p}}^{2}=0.58$, and block, $F(9$, $69.76)=30.42, p<.001, \eta_{\mathrm{p}}{ }^{2}=0.62$, on RMSE. Post hoc analysis found that the fixed gain group had a nonsignificant $(p=.90)$ difference in RMSE across days but that there was a significant decrease over practice days for the intermediate and high visual scaling groups $(p<$ .001). Post hoc analysis of the block effect included equally spaced comparisons of Block 1, Block 4, Block 7 , and Block 10 of practice. The pairwise comparisons revealed that RMSE was significantly higher on Block 1 (all $p$ values $<.01$ ). Block 4 RMSE was significantly higher than Block $7(p<.01)$ and Block $10(p<.01)$. No statistical difference was found between Blocks 7 and 10 on $\operatorname{RMSE}(p=.38)$.

The block $\times$ visual scaling group interaction failed to reach statistical significance $(p=.75)$; however, we were interested in whether the effect of visual scaling influenced the amount of change of RMSE from early (Block 1) to late (Block 10) practice. Table 1 shows the result of an effect size analysis (Cohen's $d$ ) that supports the statistical results of the practice block effect reported above and also illustrates that individuals

Table 1 Early (Block 1) and late (Block 10) practice means $\left(S D^{\mathrm{a}}\right)$, and effect size data (Cohen's $d$ ) of RMSE as a function of practice condition

\begin{tabular}{llll}
\hline & Block 1 & Block 10 & $d$ \\
\hline No Scaling & $0.273(.0714)$ & $0.220(0.054)$ & 0.72 \\
Intermediate-frequency scaling & $0.225(0.053)$ & $0.176(0.025)$ & 1.61 \\
High-frequency scaling & $0.279(0.070)$ & $0.222(0.050)$ & 0.94 \\
\hline
\end{tabular}

${ }^{\text {a }} S D=$ standard deviation 
in the intermediate frequency scaling condition exhibited the greatest reduction in task error followed by the high scaling and fixed gain conditions, respectively. The low number of subjects and unequal group distribution recruited for the experiment could be an issue for statistical power and reliability. However, given the effect size reported in Table 1, the statistical power of the practice effect was 0.90. Also, the RMSE results displayed in Fig. 2 suggest the potential for initial group difference. To examine whether there were preexisting group differences in force tracking performance RMSE of Block 1 was analyzed in a one-way ANOVA. The results of this analysis revealed nonsignificant differences $(p=.52)$ between the group for Block 1 performance.

Spectral slope Figure 3 illustrates the mean spectral slope as a function of practice for each visual scaling group. There were significant main effects of day, $F(1,19)=15.10, p<$ $.01, \eta_{\mathrm{p}}{ }^{2}=0.44$, and block, $F(9,51.29)=11.58, p<.001$, $\eta_{\mathrm{p}}{ }^{2}=0.38$, on spectral slope. The day $\times$ visual scaling group interaction, $F(2,19)=3.63, p<.05, \eta_{\mathrm{p}}{ }^{2}=0.27$, was also significant. Post hoc comparison revealed that spectral slope was significantly flatter $(\beta=-2.31)$ on Day 2 compared to Day $1(\beta=-2.54)$. Post hoc analysis of the block effect revealed that spectral slope in the early practice block was significantly steeper compared to middle and late practice phases $(p<.01)$. The fixed gain group did not show a significant change in spectral slope $(p=.79)$ from Day 1 to Day 2; however, the intermediate and high frequency scaling groups did show a significant change (flattening) in spectral slope $(p<.001$ and $p<.05$, respectively).

Coherence Figure 4 shows coherence as a function of $2 \mathrm{~Hz}$ frequency bandwidths, practice, and visual scaling condition. Overall, the results showed practice effects in the lower half of the frequency bandwidth (i.e., $0-6 \mathrm{~Hz}$ ) and significant

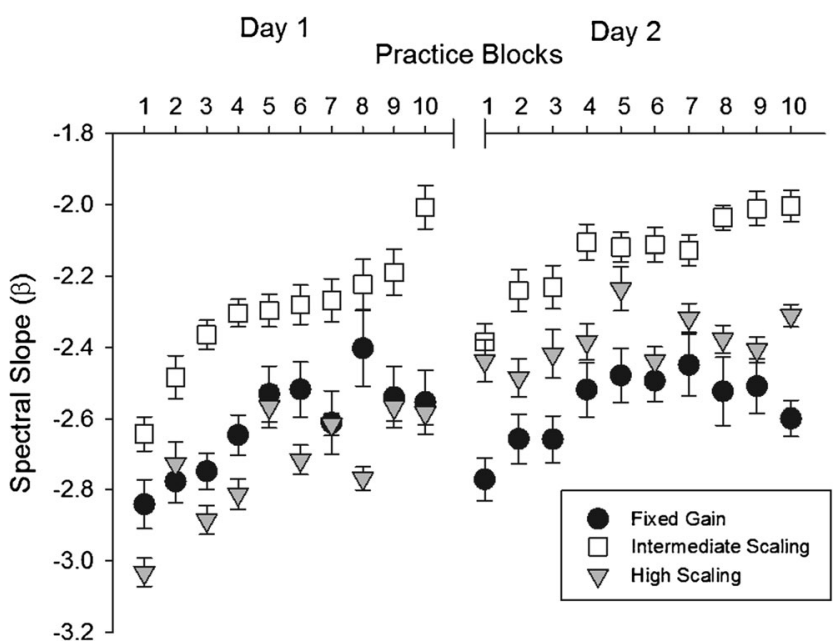

Fig. 3 Spectral slope as a function of practice and visual scaling conditions. Error bars represent standard error across participants differences between the three visual scaling conditions across the entire bandwidth of frequency structures.

In the $0-2 \mathrm{~Hz}$ bandwidth, there were significant main effects of day, $F(1,19)=10.31, p<.01, \eta_{\mathrm{p}}{ }^{2}=0.38$, block, $F(9$, $76.23)=10.45, p<.001, \eta_{\mathrm{p}}{ }^{2}=0.38$, and visual scaling, $F(2$, $19)=4.87, p<.05, \eta_{\mathrm{p}}{ }^{2}=0.36$. The $0-2 \mathrm{~Hz}$ bandwidth showed significantly lower coherence on Day $2(M=0.88, S D=$ $0.009)$ compared to Day $1(M=0.90, S D=0.014)$. Post hoc comparisons of the block (e.g., early, mid, and late) effect revealed higher coherence in early practice compared to middle and late practice phases $(p<.01)$, but no significant difference between middle to late practice $(p=.67)$. The intermediate scaling condition $(M=0.88, S D=0.019)$ exhibited significantly lower $0-2 \mathrm{~Hz}$ coherence than the fixed $(M=$ $0.90, S D=0.023)$ and high scaling $(M=0.90, S D=0.02)$ conditions ( $p<.05$ and .01 , respectively), with no difference between the latter groups.

The $2-4 \mathrm{~Hz}$ bandwidth showed a significant main effect of block, $F(9,171)=4.01, p<.01, \eta_{\mathrm{p}}{ }^{2}=0.19$. Post hoc analysis revealed lower coherence during middle and late compared to early practice phases $(p<.01)$. There was also a significant group effect, $F(2,19)=4.45, p<.05, \eta_{\mathrm{p}}{ }^{2}=0.34$ with lower coherence for the intermediate scaling condition compared to both fixed gain and high scaling conditions ( $p \mathrm{~s}<.05$ and .01 , respectively).

In the 4-6 Hz bandwidth a significant block $\times$ group interaction, $F(9,65.55)=2.08, p=.05, \eta_{\mathrm{p}}{ }^{2}=0.20$, was found on coherence. Post hoc analysis of the interaction revealed that over early, middle, and late practice phases that $4-6 \mathrm{~Hz}$ coherence decreased for the intermediate scaling group, increased for the high scaling, and remained the same for the fixed gain groups.

In the higher frequency bandwidths, there were no effects of practice (i.e., day and block) on coherence; however, significant group effects were found in the $6-8 \mathrm{~Hz}, F(2,19)=$ $12.54, p<.001, \eta_{\mathrm{p}}{ }^{2}=0.60$, and $10-12 \mathrm{~Hz}, F(2,19)=$ $13.30, p<.001, \eta_{\mathrm{p}}{ }^{2}=0.58$, frequency bandwidths. Post hoc analysis revealed that 6-8 Hz coherence of the intermediate scaling was significantly lower compared to fixed gain $(p<.001)$ and high scaling conditions $(p<.001)$. In the 10 $12 \mathrm{~Hz}$ bandwidth, post hoc comparisons revealed significantly higher coherence for the intermediate scaling group compared to fixed gain $(p<.001)$ and high scaling condition $(p<.01)$.

\section{Transfer}

RMSE The left-hand side of Fig. 5 shows the mean RMSE during the transfer tests as a function of day, transfer condition (fixed gain and $8-12 \mathrm{~Hz}$ scaling), and visual scaling group. There was a significant triple (day $\times$ transfer condition $\times$ visual scaling group) interaction, $F(2,19)=3.71, p<.05, \eta_{\mathrm{p}}{ }^{2}=$ 0.61 , on RMSE. Post hoc analysis of the three-way interaction was conducted with separate two-way (day $\times$ visual scaling 

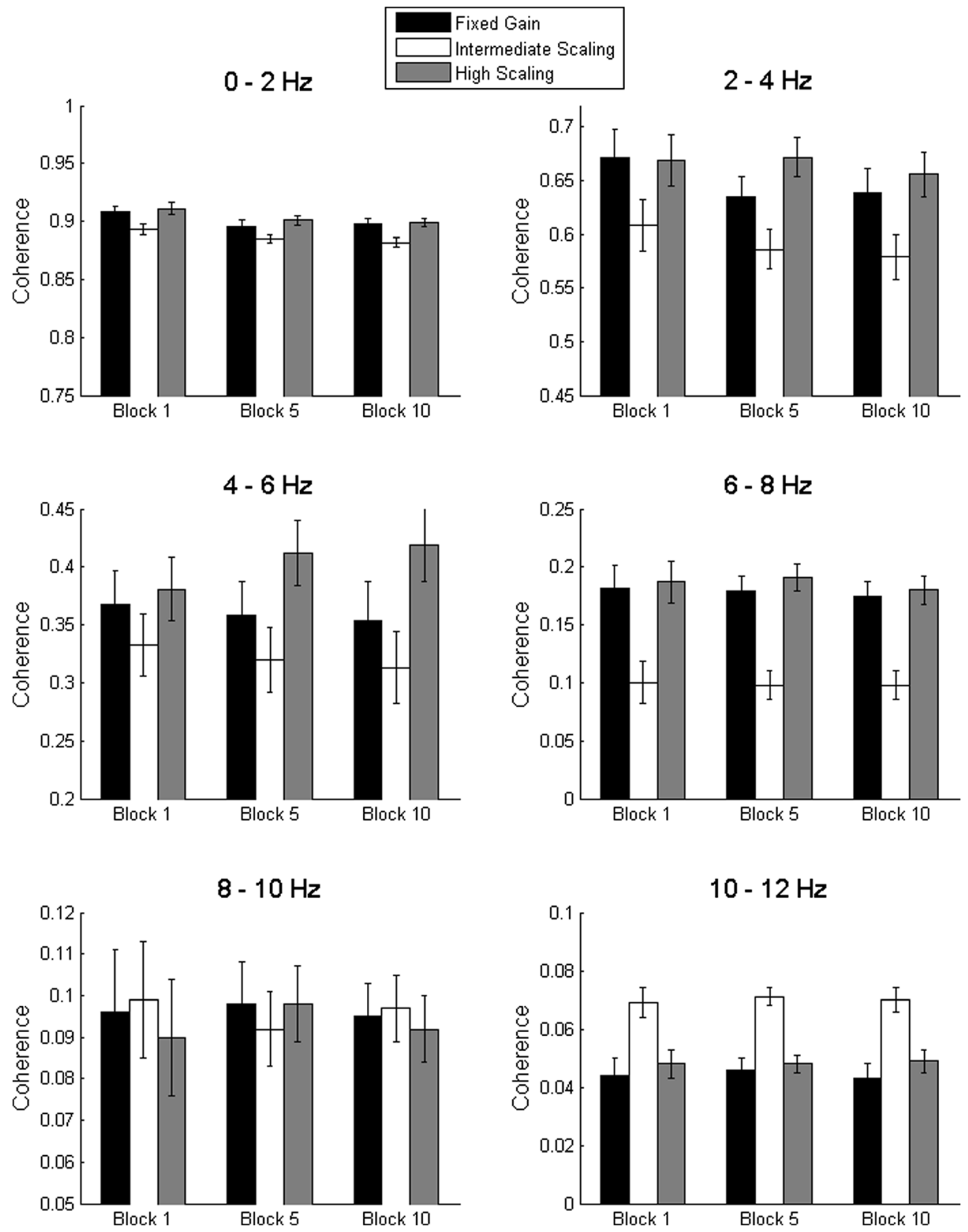

Fig. 4 Coherence between target and force output signals as a function of $2 \mathrm{~Hz}$ frequency bandwidths and visual scaling conditions

group) ANOVA for Fixed Gain and High Frequency Scaling transfer conditions. In the Fixed Gain transfer condition, a significant day $\times$ group interaction was found, $F(2,19)=$ $3.75, p<.05, \eta_{\mathrm{p}}{ }^{2}=0.28$. Post hoc analysis revealed a significant difference in RMSE between days for the intermediate $(p$ $<.05)$ and high scaling $(p<.01)$ groups but not for the fixed gain group $(p=.31)$. The results of the two-way ANOVA for the High Frequency transfer condition also revealed a significant day $\times$ group interaction, $F(2,19)=5.91, p<.01, \eta_{\mathrm{p}}{ }^{2}=$ 0.38 , on RMSE. A similar pattern of post-hoc results was observed in the High Frequency transfer condition. The intermediate $(p<.01)$ and high scaling $(p<.01)$ groups significantly reduced RMSE from Day 1 to Day 2, but the fixed gain group showed a nonsignificant difference $(p=.81)$.

Spectral slope The right-hand side of Fig. 5 illustrates the mean spectral slope $(\beta)$ as a function of day, transfer condition, and visual scaling group. There was a significant main effect of visual scaling group, $F(2,19)=3.50, p<.05, \eta_{\mathrm{p}}{ }^{2}=$ 0.28 , on spectral slope. Post hoc comparisons showed that the intermediate scaling group exhibited significantly broader 


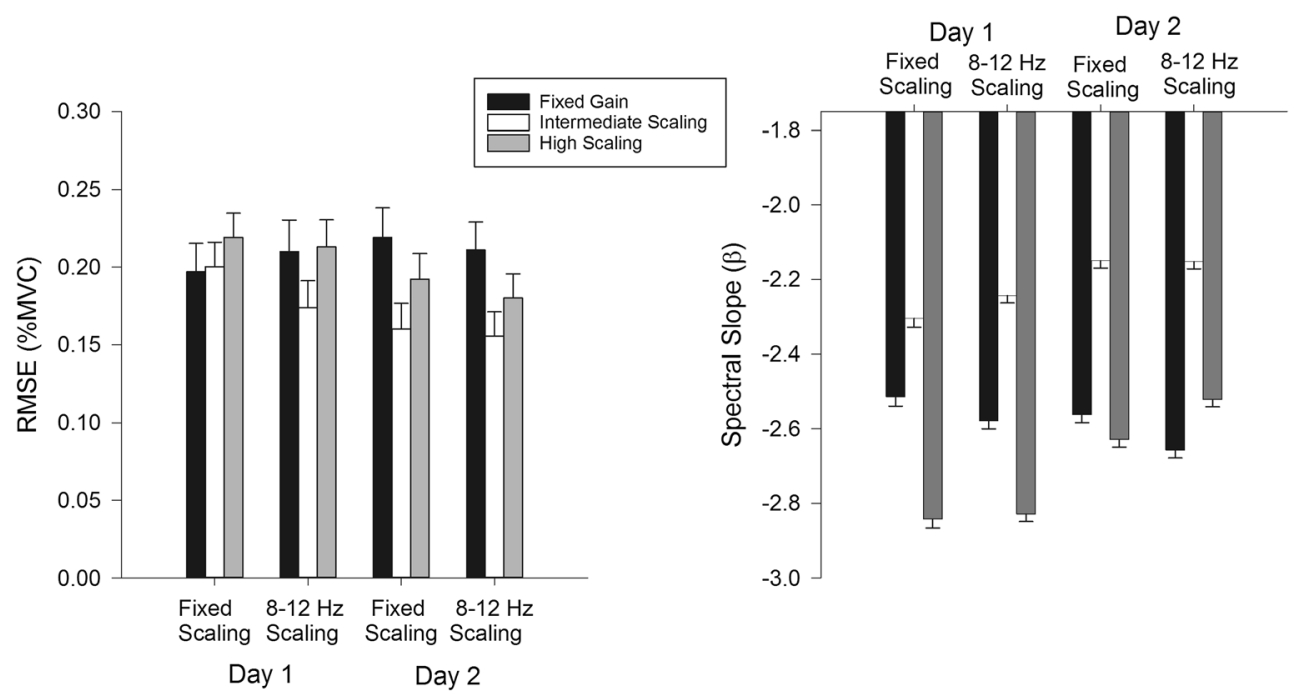

Fig. 5 RMSE (left) and spectral slope (right) as a function of day and transfer condition (i.e., no- and 8-12 Hz scaling for the three visual scaling practice groups). Error bars represent standard error across participants

spectral slopes compared to the high scaling $(p<.05)$ and fixed gain $(p<.01)$ groups.

The effectiveness of the visual scaling manipulation was dependent on the presence of higher frequency components (i.e., broader spectral slope) in the force output. Also, individual differences in the organization of the force frequency structures potentially influences the visual feedback information presented to participants. To examine the influence of the selective visual scaling manipulation on task performance, a correlation analysis was computed across all participants between spectral slope ( $\beta$ ) and task error (RMSE) in the $8-12 \mathrm{~Hz}$ scaling transfer test. Figure 6 shows a significant $(p<.001)$ inverse relation $(r=-0.65)$ in that individuals with broader spectral slopes exhibited lower task error compared to individuals with steeper spectral slopes.

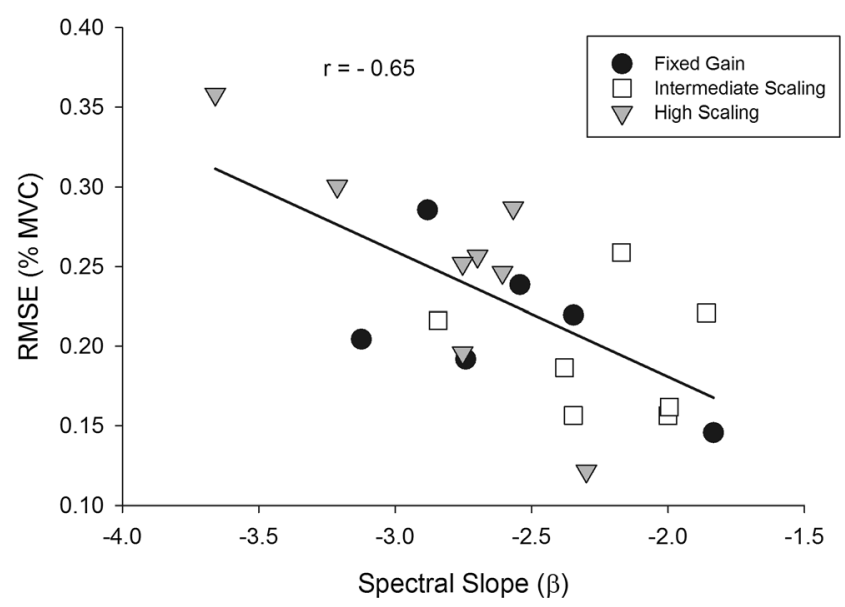

Fig. 6 Correlation between spectral slope and RMSE during the $8-12 \mathrm{~Hz}$ visual scaling transfer condition across all participants
The same correlation analysis was also conducted for the Fixed Gain transfer condition. The results showed a nonsignificant relation between spectral slope and task error $(p=$ .59). The different patterns of correlation suggest that individuals used the augmented faster time scales of visual information to achieve higher levels of performance relative to the task goal but this ability was isolated to the high frequency visual scaling transfer condition.

\section{Discussion}

The current experiment was set up to investigate the learning and generalization of isometric force dynamics as a function of practice and selective scaling of visual feedback information (Marteniuk \& Romanow, 1983; Sosnoff et al., 2009; Stanley \& Franks, 1990). Three experimental groups practiced an isometric force-tracking task to an irregular target pattern under different visual feedback constraints - fixed gain, intermediate $(4-8 \mathrm{~Hz})$, or high $(8-12 \mathrm{~Hz})$ selective scaling conditions - over two consecutive practice days. At the end of each practice day, two transfer tests were administered to assess transfer of learning. The principal questions were whether (a) practice would differentially alter the multiple time scales of force output as well as interact with the visual scaling manipulation (Hu \& Newell, 2010); (b) the selective visual scaling manipulation would generalize across the multiples time scale of force output in a manner consistent with fractal models of physiology and behavior (Bassingthwaighte et al., 1994; Ward, 2002); and (c) the selective visual scaling manipulation of the practice environment would induce enhanced transfer to novel task conditions compared to the fixed gain group. 


\section{Practice and force output structure}

Previous studies have shown bidirectional changes (Newell \& Vaillancourt, 2001) and differential time scales of learning (Studenka et al., 2014) of isometric force dynamics as a function of practice. Here, task outcome (RMSE) showed reductions of error over practice (block and day) as well as an interaction between practice and visual scaling conditions. Over the 2 practice days, RMSE was lower for the selective visual scaling conditions (intermediate and high) compared to the fixed gain group. Moreover, the selective scaling groups showed a persistent level of performance following a $24 \mathrm{hr}$ period of rest - a finding that was not evident for the fixed gain group. These results suggest that the selective scaling of visual information facilitated the retention of the faster time scales of force output.

Reduction in tracking error was aided by the reorganization of force output dynamics over practice and was assessed through frequency domain analyses of spectral slope and coherence. A comparison of the spectral slope of force output (and it change over practice) with that of the target pattern indicates an individual's adaptation of force output regularity

(Bassingthwaighte et al., 1994). All individuals showed the expected task-dependent shift of the spectral slope that approached, but did not exactly match, the frequency profile of the target pattern (see Fig. 3). At the end of each practice day the intermediate visual scaling group exhibited the flattest spectral slope followed by the high visual scaling and fixed gain groups, respectively.

The correlation between force output and the target pattern in the frequency domain showed a general reduction of coherence as a function of frequency bandwidth and practice. Small effects of practice were evident in the lower frequency bandwidths $(0-6 \mathrm{~Hz})$, but the influence of the selective visual scaling manipulation was observed across most frequency bandwidths. In particular, the intermediate scaling group showed lower coherence over the middle range of frequency components $(4-8 \mathrm{~Hz})$ and higher coherence in the $8-12 \mathrm{~Hz}$ bandwidths compared to the other groups. Consistent with the findings of Hu and Newell (2010), task performance and force adaptability in the $8-12 \mathrm{~Hz}$ manipulation showed a lower degree of effectiveness compared to the $4-8 \mathrm{~Hz}$ scaling. These findings indicate that a change in one time scale of force output via practice or under visual gain manipulations induces changes across the multiple time scales of force control that are consistent with self-organization properties of motor behavior (Haken, 1983; Van Orden, Holden, \& Turvey, 2003).

Continuous motor tasks (i.e., bimanual coordination and isometric force tracking) afford the potential to capture a broader range of time scales of change than the typical discrete movement used in most motor learning investigations. They, also, allow for the examination of properties of learning over multiple dimensions of movement (Studenka et al., 2014) to potentially reveal how the perceptual-motor system learns and retains successful movement patterns with redundant motor tasks. The observed persistence of tracking performance under the visual scaling manipulation represents a novel finding for this isometric force tracking task and suggests that augmenting the shorter time scales of visual feedback information of force output exerts a stronger influence on the learning and retention of force output than that of traditional practice structure manipulations including variability of practice (Schmidt, 1975).

\section{Selective visual scaling and $\mathbf{1} / \mathbf{f}$ structure}

A second question addressed the interaction between selective scaling manipulation and practice on the organization of force output. Specifically, we investigated the hypothesis that the intermediate and high visual scaling groups would show enhanced tracking performance through a force control strategy that included greater relative contributions of shorter time scales. Overall, the effect of visual scaling was greatest for the intermediate (4-8 Hz) bandwidth, but the high visual scaling group (8-12 Hz) also exhibited a progressive reduction in task error as a function of practice; albeit, at a slower rate of change than the intermediate scaling condition - a finding that is consistent with the notion of multiple dimensions of motor learning (Mayer-Kress, Deutsch, \& Newell, 2003; Newell, Liu, \& Mayer-Kress, 2001).

Reduction in tracking error under the selective scaling manipulation was achieved via a collective reorganization of slow and fast time scales of force output. This manipulation is distinctly different to traditional visual gain approaches that equally enhance the relative visual feedback information of all frequency components (Jagacinski \& Flach, 2003). Also, it is important to note that the characteristic properties of slow (high amplitude movements) and fast (low amplitude oscillations) time scales constrain the adaptive nature of isometric force control. Collectively, the findings support the notion that force output exhibits a modifiable bandwidth of frequency components that extends to $\sim 12 \mathrm{~Hz}$. Additional constraints, such as limits to the processing capacity of faster time scales of visual information (Carlton, 1992) or the presence of physiological tremor (Elble \& Koller, 1990), may be limiting factors to the adaptive nature of isometric force control but further investigation is needed to address such issues. Overall, the adaptive shifts in short and long time scales of force output under the selective scaling manipulation supports the interdependence notion between multiple times scales of motor output (Haken, 1983; Mayer-Kress et al., 2003; Van Orden et al., 2003).

\section{Informational constraints and transfer}

The current investigation examined two forms of generalization - generalization of the effect of selective visual scaling 
across the multiple time scales of force control and whether the practice environments differentially transferred to novel visual scaling conditions. The latter issue relates to the traditional issue of transfer of motor learning (Bilodeau \& Bilodeau, 1961), while the former addresses interdependence between the multiple time scales of force control (Haken, 1983; Van Orden et al., 2003)

Evidence has provided strong support that human motor behavior in tasks such as temporal estimation (Gilden, 1997) and locomotion (Hausdorff et al., 1997) exhibit selforganizing properties associated with $1 /$ f models of motor control. However, there has been limited study of such systems from a motor learning perspective. From a motor control perspective, isometric force tracking performance is reflected by the ability to modulate slow and fast time-scale processes. Slower time scales are typically taken to reflect traditional performance variables related to higher level cognitive processes while faster time scales relate to predictive, feedforward processes of self-organization (Mayer-Kress et al., 2003). A key question that requires further exploration is how these oscillatory time scales of force control interact with the growth/decay time scales of learning and development (Newell et al., 2001). The current findings provide further evidence that the oscillatory time scales of motor output show different characteristics of growth/decay time scales of learning (Studenka et al., 2014).

According to Haken (1983) and Van Orden, Hollis, and Wallot (2012), slower time scales reflecting circadian rhythms and persistence of behavioral change constrain the adaptive ability to modulate faster, oscillatory time scales of motor output. Studenka et al. (2014) recently found support for this idea in revealing a lack of persistence of faster time scales of isometric force production over multiple days of practice. The present findings, however, showed that practicing isometric force control under specific constraints (i.e., selective scaling of visual feedback information) enhanced the learned adaptability of force output structure. It is not clear whether a slow-to-fast (Haken, 1983; Van Orden et al., 2012) or fast-to-slow direction occurred in terms of learning to use the multiple time scales of force control but the findings clearly demonstrate that the selective visual scaling of shorter time scales of visual information altered the interaction between multiple time scale processes of force control and led to a different time scale of learning (change over time).

In motor learning the practice environment and previous experience can have a significant influence on performance in novel motor tasks. According to the specificity of practice hypothesis (Proteau, Marteniuk, \& Levesque, 1992; Tremblay \& Proteau, 1998), transfer conditions that change the dominant source of afferent information used during practice to ensure optimal performance often degrades performance. Thus, it could be predicted here that the two transfer conditions that altered (added or removed) the augmented visual information of faster time scales of force output would result in increased tracking error. However, in the constraints approach, the selective scaling of visual feedback information channels individuals to explore different movement solutions that may not be possible under normal gain conditions. Theoretically, performance in the transfer conditions is dependent an individual's ability to map information and movement dynamics in the perceptual-motor workspace.

The transfer results did not support the specificity of practice hypothesis in that the intermediate scaling group exhibited lower tracking error compared to the high scaling and fixed gain groups in both transfer conditions even though the visual feedback information was not specific to their practice environment. The significant day by group interaction found in both transfer conditions showed that the intermediate and high scaling groups exhibited lower tracking error than the fixed gain group. Overall, the transfer findings show that the augmented visual information of intermediate frequency components (4$8 \mathrm{~Hz}$ ) during practice channeled individuals toward relevant perceptual information that not only facilitated tracking performance but also transferred to novel feedback condition (see Bennett et al., 1999; Fowler \& Turvey, 1978). Additional support for the constraints approach was found in the significant correlation between the spectral slope and task error in the 8$12 \mathrm{~Hz}$ transfer condition (see Fig. 6). The inverse relation between spectral slope and task error (see Fig. 6) suggests that reduction in tracking error was in part dependent on the ability to adaptively modulate faster time scales of force output.

In summary, the current study found that the interaction of practice and selective visual information scaling broadened the adaptive range of force output dynamics and enhanced the retention and transfer of practice-induced changes following periods of rest. Individuals showed a task-dependent ability to modulate the relative shorter time scales of force output dynamics that was greater in the intermediate $(4-8 \mathrm{~Hz})$ and high $(8-12 \mathrm{~Hz})$ visual scaling conditions compared to the standard fixed gain, and this practice effect persisted in novel transfer conditions.

Collectively, the findings exemplify signature features of fractal models of behavioral output that have multiple time scales of control (Bassingthwaighte et al., 1994; MayerKress et al., 2003; Van Orden et al., 2003) and highlights the need to understand the multiple dimensions of learning that extend beyond standard motor learning performance outcome variables.

\section{References}

Armstrong, T. R. (1970). Feedback and perceptual-motor skill learning: A review of information feedback and manual guidance training techniques 
Bassingthwaighte, J. B., Liebovitch, L. S., \& West, B. J. (1994). Fractal physiology. New York: Oxford University Press.

Benesty, J., Chen, J. D., Huang, Y. T. A., \& Member, S. (2005). A generalized MVDR spectrum. IEEE Signal Processing Letter, 12(12), 827-830.

Bennett, S., Davids, K., \& Woodcock, J. (1999). Structural organization of practice: Effects of practicing under different informational constraints on the acquisition of one-handed catching skills. Journal of Motor Behavior, 31(1), 3-9.

Beuter, A., Haverkamp, H., Glass, L., \& Carriére, L. (1995). Effect of manipulating visual feedback parameters on eye and finger movements. International Journal of Neuroscience, 83, 281.

Bilodeau, E. A., \& Bilodeau, I. M. (1961). Motor-skills learning. Annual Review of Psychology, 12(1), 243-280.

Carlton, L. G. (1992). Visual processing time and the control of movement. In L. Proteau \& D. E. Elliott (Eds.), Vision and motor control: Advances in psychology (pp. 3-31). New York: Elsevier.

Elble, R. J., \& Koller, W. C. (1990). Tremor. Baltimore: John Hopkins University Press.

Ernst, M. O., \& Banks, M. S. (2002). Humans integrate visual and haptic information in a statistically optimal fashion. Nature, 415(6870), 429-433. doi:10.1038/415429a

Fowler, C. A., \& Turvey, M. T. (1978). Skill acquisition: An event approach with special reference to searching for the optimum of a function of several variables. In G. E. Stelmach (Ed.), Information processing in motor control. New York: Academic Press.

Gilden, D. L. (1997). Fluctuations in the time required for elementary decisions. Psychological Science, 8(4), 296-301. doi:10.1111/j. 1467-9280.1997.tb00441.x

Haken, H. (1983). Synergetics: An introduction (3rd ed.). Berlin: Springer-Verlag.

Hausdorff, J. M., Mitchell, S. L., Firtion, R., Peng, C. K., Cudkowicz, M. E., Wei, J. Y., ... Jeffrey, M. (1997). Altered fractal dynamics of gait: Reduced stride-interval correlations with aging and Huntington's disease. Journal of Applied Physiology, 82(1), 262-269

Hodges, N. J., \& Franks, I. M. (2004). Instruction, demonstrations, and the learning process. In A. M. Williams \& N. J. Hodges (Eds.), Skill Acquisition in sport: Research, theory and practice (pp. 145-174). London: Routledge.

Hong, S. L., \& Newell, K. M. (2008). Visual information gain and the regulation of constant force levels. Experimental Brain Research, 189(1), 61-69. doi:10.1007/s00221-008-1403-Z

Hu, X., \& Newell, K. M. (2010). Adaptation to selective visual scaling of short time scale processes in isometric force. Neuroscience Letters, 469(1), 131-134. doi:10.1016/j.neulet.2009.11.059

Jagacinski, R. J., \& Flach, J. (2003). Control theory for humans: Quantitative approaches to modeling performance. Danvers: CRC Press.

Kernodle, M. W., \& Carlton, L. G. (1992). Information feedback and the learning of multiple degree of freedom activities. Journal of Motor Behavior, 24(2), 187-195.

King, A. C., \& Newell, K. M. (2013). The learning of isometric force time scales is differentially influenced by constant and variable practice. Experimental Brain Research, 227(2), 149-159. doi:10.1007/ s00221-013-3446-Z

Kugler, P. N., Kelso, J. A. S., \& Turvey, M. T. (1980). On the concept of coordinative structures as dissipative structures: I. Theoretical lines of convergence. Tutorials in Motor Behavior, 3, 47.

Marteniuk, R. G., \& Romanow, S. K. E. (1983). Human movement organization and learning as revealed by variability of movement, use of kinematic information and Fourier analysis. In R. A. Magill (Ed.), Memory and control of action (pp. 167-197). Amsterdam, The Netherlands, North-Holland

Mayer-Kress, G., Deutsch, K. M., \& Newell, K. M. (2003). Modeling the control of isometric force production with piece-wise linear, stochastic maps of multiple time-scales. Fluctuation and Noise Letters, 3(1), L123-L129.
Newell, K. M. (1991). Motor skill acquisition. Annual Review of Psychology, 42, 213-237.

Newell, K. M. (1996). Change in movement and skill: Learning, retention, and transfer. In M. L. Latash \& M. T. Turvey (Eds.), Dexterity and its development (pp. 393-429). Hillsdale: Erlbaum.

Newell, K. M., Broderick, M. P., Deutsch, K. M., \& Slifkin, A. B. (2003). Task goals and change in dynamical degrees of freedom with motor learning. Journal of Experimental Psychology: Human Perception and Performance, 29(2), 379-387. doi:10.1037/0096-1523.29.2.379

Newell, K. M., Liu, Y. T., \& Mayer-Kress, G. (2001). Time scales in motor learning and development. Psychological Review, 108(1), 57-82.

Newell, K. M., \& McDonald, P. V. (1994). Information, coordination modes, and control in a prehensile force task. Human Movement Science, 13, 375-392.

Newell, K. M., Morris, L. R., \& Scully, D. M. (1985). Augmented information and the acquisition of skill in physical activity. Exercise and Sport Sciences Reviews, 13(1), 235-262.

Newell, K. M., \& Vaillancourt, D. E. (2001). Dimensional change in motor learning. Human Movement Science, 20(4), 695-715. doi: 10.1016/S0167-9457(01)00073-2

Pew, R. W. (1974). Levels of analysis in motor control. Brain Research, 71(2-3), 393-400. doi:10.1016/0006-8993(74)90983-4

Poulton, E. C. (1974). Tracking skill and manual control. New York: Academic Press.

Proteau, L. (1992). On the specificity of learning and the role of visual information for movement control. In L. Proteau \& D. E. Elliott (Eds.), Vision and motor control (pp. 67-104). New York: Elsevier.

Proteau, L., Marteniuk, R. G., \& Levesque, L. (1992). A sensorimotor basis for motor learning: Evidence indicating specificity of practice. Quarterly Journal of Experimental Psychology, Section A: Human Experimental Psychology, 44(3), 557-575.

Proteau, L., Tremblay, L., \& Dejaeger, D. (1998). Practice does not diminish the role of visual information in on-line control of a precision walking task: Support for the specificity of practice hypothesis. Journal of Motor Behavior, 30(2), 143-150.

Schmidt, R. A. (1975). A schema theory of discrete motor skill learning. Psychological Review, 82(4), 225-260.

Slifkin, A. B., Vaillancourt, D. E., \& Newell, K. M. (2000). Intermittency in the control of continuous force production. Journal of Neurophysiology, 84(4), 1708-1718.

Sosnoff, J. J., \& Newell, K. M. (2006). Information processing limitations with aging in the visual scaling of isometric force. Experimental Brain Research, 170(3), 423-432. doi:10.1007/ s00221-005-0225-5

Sosnoff, J. J., \& Newell, K. M. (2008). Age-related loss of adaptability to fast time scales in motor variability. Journals of Gerontology. Series B, Psychological Sciences and Social Sciences, 63(6), 344-352.

Sosnoff, J. J., Valantine, A. D., \& Newell, K. M. (2009). The adaptive range of $1 / \mathrm{f}$ isometric force production. Journal of Experimental Psychology: Human Perception and Performance, 35(2), 439446. doi:10.1037/a0012731

Sosnoff, J. J., \& Voudrie, S. J. (2009). Practice and age-related loss of adaptability in sensorimotor performance. Journal of Motor Behavior, 41(2), 137-146. doi:10.3200/JMBR.41.2.137-146

Stanley, M. L., \& Franks, I. M. (1990). Learning to organize the frequency components of a perceptual motor skill. Human Movement Science, 9, 291-323.

Studenka, B. E., King, A. C., \& Newell, K. M. (2014). Differential time scales of change to learning frequency structures of isometric force tracking. Journal of Experimental Psychology: Human Perception and Performance, 40(4), 16-29. 
Studenka, B. E., \& Newell, K. M. (2013). Visual information for prospective control of tracking irregular target paths with isometric force production. Journal of Experimental Psychology: Human Perception and Performance, 39(6), 1557-1567. doi:10.1037/ a0031744

Swinnen, S. P., Schmidt, R. A., Nicholson, D. E., \& Shapiro, D. C. (1990). Information feedback for skill acquisition: Instantaneous knowledge of results degrades learning. Journal of Experimental Psychology: Learning, Memory, and Cognition, 16(4), 706-716.

Tremblay, L., \& Proteau, L. (1998). Specificity of practice: The case of powerlifting. Research Quarterly for Exercise and Sport, 69(3), 284-289. doi:10.1080/02701367.1998.10607695
Van Orden, G. C., Holden, J. G., \& Turvey, M. T. (2003). Self-organization of cognitive performance. Journal of Experimental Psychology: General, 132(3), 331-350. doi:10.1037/0096-3445.132.3.331

Van Orden, G. C., Hollis, G., Wallot, S., \& Orden, G. V. (2012). The bluecollar brain. Frontiers in Physiology, 3, 207. doi:10.3389/fphys. 2012.00207

Ward, L. M. (2002). Dynamical cognitive science. Cambridge: MIT Press.

Whiting, H. T. A., Savelsbergh, G. J. P., \& Pijpers, J. R. (1995). Specificity of motor learning does not deny flexibility. Applied Psychology, 44(4), 315-332. doi:10.1111/j.1464-0597.1995. tb01083.x 